\title{
Current epidemiology of low back pain
}

\author{
Camilla Mattiuzzi ${ }^{1}$, Giuseppe Lippi ${ }^{2}$, Chiara Bovo ${ }^{3}$ \\ ${ }^{1}$ Service of Clinical Governance, Provincial Agency for Social and Sanitary Services, Trento, Italy; ${ }^{2}$ Section of Clinical Biochemistry, University of \\ Verona, Verona, Italy; ${ }^{3}$ Medical Direction, University Hospital of Verona, Verona, Italy \\ Contributions: (I) Conception and design: All Authors; (II) Administrative support: None; (III) Provision of study materials or patients: None; (IV) \\ Collection and assembly of data: G Lippi; (V) Data analysis and interpretation: All Authors; (VI) Manuscript writing: All authors; (VII) Final approval \\ of manuscript: All authors. \\ Correspondence to: Prof. Giuseppe Lippi. Section of Clinical Biochemistry, University Hospital of Verona, Piazzale LA Scuro, 37134 Verona, Italy. \\ Email: giuseppe.lippi@univr.it.
}

Background: Low back pain (LBP) is a major disabling pathology, whose clinical, societal and economic burden remains mostly overlooked. Therefore, we aim to provide here a concise overview on the recent worldwide epidemiology of this condition.

Methods: An electronic search was performed in the Global Health Data Exchange (GHDx) repository, a large worldwide database of health-related data, using the keyword "low back pain". The result of our electronic search was downloaded in comma-separated values (CSV), imported into an Excel file and analyzed.

Results: The current estimates of incidence, prevalence and disability-adjusted life years (DALYs) of LBPs are 245.9 million cases/year $\left(3.2 \% ; 15^{\text {th }}\right.$ worldwide cause), 577.0 million cases $\left(7.6 \% ; 15^{\text {th }}\right.$ worldwide cause) and 64.9 million DALYs (2.6\% of all DALYs; $6^{\text {th }}$ worldwide cause), respectively. All these measures displayed a considerable $\sim 50 \%$ increase during the last 20 years. The burden of all LBP cases is marginally higher in women than in men, exhibits gradual increase from the birth age, reaching the peak between 40-50 years, and then progressively declining. An analysis within each age range shows that prevalence of LBP among all human diseases grows in parallel with ageing, with the most notable increase after 80 years. The risk of LBD increases in parallel with socio-demographic index (SDI), being over 3-fold higher in high than in low SDI countries. According to a linear fit based on data of the last 20 years, incidence, prevalence and DALYs of LBP may further increase by $\sim 1.4$ fold by the year 2050 .

Conclusions: LBP poses a significant worldwide epidemiologic burden, especially in women, adults and high SDI countries, displaying an escalating trend that is not expected to reverse soon.

Keywords: Low back pain (LBP); disability; epidemiology; statistics

Received: 23 January 2020. Accepted: 03 April 2020; Published: 25 June 2020.

doi: 10.21037/jhmhp-20-17

View this article at: http://dx.doi.org/10.21037/jhmhp-20-17

\section{Introduction}

Low back pain (LBP) is conventionally defined as pain, muscle tension or stiffness localized below the costal margin and above the inferior gluteal folds, with or without associated leg pain (1). LBP is conventionally classified as non-specific (i.e., up to $90 \%$ of cases, when the physiopathological source cannot be identified) or specific, the latter cases mostly due to hernias, fractures osteoporosis, rheumatic diseases, spondyloarthropathy, infections or cancer. Markers of possible root problems encompass irradiation to foot or toes, onset of numbness and/or paraesthesia, leg pain triggered by straight leg raising and localised neurology (e.g., circumscribed to a single nerve root). LBP can also be classified as acute (i.e., lasting $<6$ weeks), sub-acute (i.e., lasting between 6 weeks and 3 months) or chronic (i.e., lasting for more than 3 months) (1). 


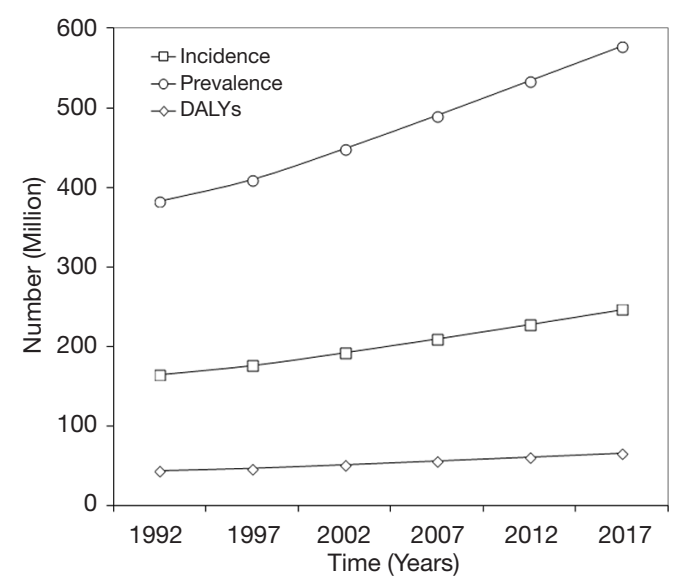

Figure 1 Worldwide incidence, prevalence and DALYs of LBP cases recorded in each age range. DALYs, disability-adjusted life years; LBP, low back pain.

Most episodes of LBP have favourable prognosis, especially when a specific cause can not be identified and the trigger cannot be directly related to severe systemic diseases such as cancer, rheumatic disorders and so forth (2).

It is generally assumed that LBP is a very common condition worldwide, occurring with a relatively high frequency in the general population, so that up to $80 \%$ individuals will experience an episode at some point throughout the lifetime (3). Information on LBP epidemiology has been published in some previous articles, but data have been mostly obtained with individual searches in scientific electronic databases such as PubMed, EMBASE and others, and may hence suffer from selection and publication bias (4-7). Even more importantly, no recent statistics has been published on the worldwide burden of LPB, at least during the last 3 years to the best of our knowledge (8). Therefore, the aim of this article is to provide a concise, updated overview on recent official data on worldwide epidemiology of LBP.

\section{Methods}

The epidemiologic information on back and neck pain was retrieved from the Global Health Data Exchange (GHDx) repository, which is a large worldwide database of health-related data maintained by the Institute for Health Metrics and Evaluation (9). This large catalogue contains information on approximately 350 human diseases, from 195 different countries and territories. The information is principally derived from survey and surveillance data, admission and outpatient records, health insurance claims and literature studies, for a total number of $\sim 69,000$ reliable sources (10).

A specific electronic search was conducted for the condition "low back pain". According to GHDx, "low back pain" was defined as LBP (with or without pain referred into one or both lower limbs) lasting for $\geq 1$ day, where low back was defined as the area on posterior aspect of the body from the lower margin of the twelfth ribs to lower gluteal folds (ICD-10 codes: M54.3, M54.4 and M54.5). The electronic search was then complemented by selecting additional epidemiologic variables, such as "measure" ("incidence" AND "prevalence" AND "DALYs", where DALYs stands for cause-specific disability-adjusted life years), sex ("male" AND "female"), "metric" ("number"), "year" ("1992" AND “1997” AND "2002” AND "2007” AND “2012”AND "2017"), "age" (“<5" AND "5 to 9" AND "10 to 14" AND "15 to 19 " AND "20 to 24 " AND " 25 to 29 " AND "30 to 34 " AND "35 to 39 " AND "40 to 44" AND "45 to 49" AND "50 to 54" AND "55 to 59" AND "60 to 64" AND "65 to 69" AND "70 to 74" AND "75 to 79" AND "80 plus") and "location" ("Low SDI" AND "Low-middle SDI" AND "Middle SDI" AND "High-middle SDI" AND "High SDI", where SDI stands for socio-demographic index). The year 2017 was selected for obtaining the most updated epidemiologic picture, whereby 2017 is the last searchable year in GHDx database. The final output of the electronic search carried out according to the previously mentioned criteria was downloaded in comma-separated values (CSV), imported into a Microsoft Excel file (Microsoft, Redmond, WA, US), and the analysis was then carried out with Microsoft Excel and MedCalc statistical software (MedCalc Software, Ostend, Belgium). The study was performed in accordance with the Declaration of Helsinki, under the terms of relevant local legislation. The data in this article were taken from a public database, so ethical review is exempt.

\section{Results}

The worldwide epidemiologic burden of LBP observed in the last 20 years, along with the current situation, are shown in Figure 1. The current incidence, prevalence and DALYs account for 245.9 million cases/year $\left(15^{\text {th }}\right.$ worldwide cause; $32.4 \%$ cases associated with leg pain), 577.0 million cases $\left(15^{\text {th }}\right.$ worldwide cause) and 64.9 million DALYs $\left(6^{\text {th }}\right.$ worldwide cause), respectively. All these epidemiologic 


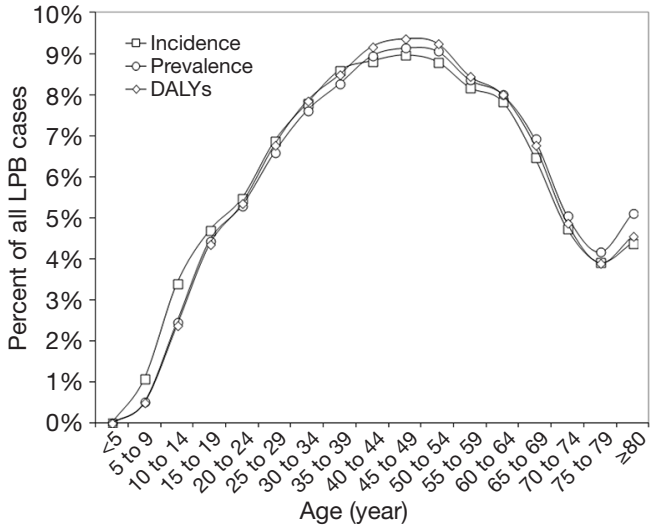

Figure 2 Percent of the total number of LBP cases recorded in each age range. DALYs, disability-adjusted life years; LBP, low back pain.

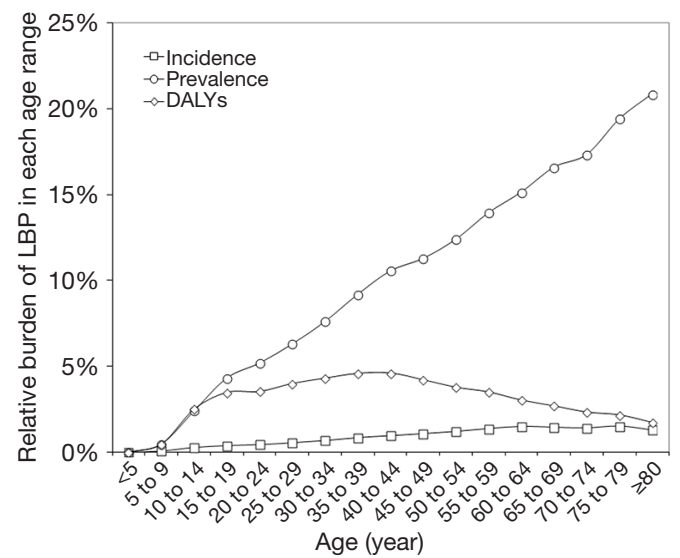

Figure 3 Relative burden of LBP within each age range. DALYs, disability-adjusted life years; LBP, low back pain.

measures displayed a remarkable increase during the last 20 years, recording an increment between $49.8-50.7 \%$, and displaying an almost linear trend $(r=0.997$ for incidence and prevalence, $\mathrm{r}=0.998$ for DALYs; all $\mathrm{P}<0.001)$. The current relative values of incidence, prevalence and DALYs of LBP are hence $0.6 \%, 7.8 \%$ and $2.6 \%$ of all causes, respectively, whilst incidence and prevalence in the worldwide population are $3.2 \%$ and $7.6 \%$, respectively. Among these, the relative burden of LBP in terms of DALYs has exhibited the sharpest increased during the last 20 years ( 1.5 -fold, from $1.67 \%$ in 1992 to $2.60 \%$ in 2017). According to GDHx data, the burden of LBP is marginally higher in women than in men. More specifically, the incidence is $0.69 \%$ versus $0.59 \%$ [odds ratio (OR), 1.16 ;
$95 \%$ confidence interval ( $95 \% \mathrm{CI}), 1.07-1.26 ; \mathrm{P}<0.001)$, the prevalence is $8.57 \%$ versus $7.09 \%$ (OR, 1.23 ; $95 \%$ CI, 1.21 1.25 ; $\mathrm{P}<0.001$ ), whilst DALYs are $3.07 \%$ versus $2.19 \%$ (OR, $1.41 ; 95 \%$ CI, $1.34-1.49 ; \mathrm{P}<0.001)$. The age distribution of incidence, prevalence and DALYs of LBP is shown in Figure 2. Cases of LBP exhibit a gradual increase from the birth age, reaching the peak between $40-50$ years, and then progressively declining. Overall, $~ 50 \%$ of all LBP cases and DALYs are recorded between 35 and 64 years. The relative values of incidence, prevalence and DALYs of LBP among all human diseases and within each age range are shown in Figure 3. The incidence and prevalence display an almost linear relationship, paralleling age, and approximating $1.8 \%$ and $21 \%$ after the age of 80 years. Unlike prevalence, DALYs display a peculiar trend, beginning to increase after the age of 10 years, gradually increasing up to the age of $40-44$ years and then progressively declining in the elderly. The burden of LBP stratified according to the sociodemographic index (SDI) is shown in Table 1. The risk of developing, and then being debilitated by LBD, increases in parallel with SDI, being over 3-fold higher in high than in low SDI countries (over 4-fold difference for DALYs). Finally, according to a linear fit based on data of the last 20 years it can be estimated that incidence, prevalence and DALYs of LBP will further increase between 1.44-1.45 folds by the year 2050 .

\section{Discussion}

The results of our analysis, based on the GHDx database, attest that the worldwide impact of LBP is relevant. Although the incidence remains relatively low (i.e., $<1 \%$ ), the values of prevalence (i.e., $~ 8 \% ; 15^{\text {th }}$ worldwide cause) and DALYs (i.e., $2.6 \%$; $6^{\text {th }}$ worldwide cause of DALYs) would contribute to classify LBP as a primary healthcare issue. Even more concerning are the projections based on the trend recorded during the last 20 years, whereby incidence, prevalence and DALYs would increase up to 353.5 million cases/year (0.70\%), 833.8 million cases $(8.3 \%)$ and 93.8 million (4.0\%), respectively. The predictable trend of DALYs is especially alarming, since such an increase from the current relative burden of LBP (i.e., from $2.6 \%$ to $4.0 \%$ ) implies that this condition will have an even more devastating impact on "healthy" life in the future. This evidence would require to make additional efforts on prevention and care of LBP, by increasing public funding on translational research focused on identifying potentially 
Table 1 Burden of low back-pain stratified according to the SDI

\begin{tabular}{|c|c|c|c|c|c|c|}
\hline SDI & \multicolumn{2}{|r|}{ Incidence } & \multicolumn{2}{|c|}{ Prevalence } & \multicolumn{2}{|r|}{ DALYs } \\
\hline Low SDI & 0.40 & - & 5.37 & - & 1.37 & - \\
\hline Low-middle SDI & 0.50 & $\begin{array}{c}1.26(95 \% \mathrm{Cl}, \\
1.20-1.32 ; \mathrm{P}<0.001)\end{array}$ & 6.32 & $\begin{array}{c}1.19(95 \% \mathrm{Cl} \\
1.15-1.23 ; \mathrm{P}<0.001)\end{array}$ & 1.84 & $\begin{array}{c}1.34(95 \% \mathrm{Cl}, \\
1.22-1.4 ; \mathrm{P}<0.001)\end{array}$ \\
\hline High-middle SDI & 0.76 & $\begin{array}{c}1.91(95 \% \mathrm{Cl}, \\
1.83-2.00 ; \mathrm{P}<0.001)\end{array}$ & 8.53 & $\begin{array}{c}1.64(95 \% \mathrm{Cl}, \\
1.59-1.69 ; \mathrm{P}<0.001)\end{array}$ & 3.24 & $\begin{array}{c}2.41(95 \% \mathrm{Cl}, \\
2.20-2.64 ; \mathrm{P}<0.001)\end{array}$ \\
\hline High SDI & 1.21 & $\begin{array}{c}3.04(95 \% \mathrm{Cl}, \\
2.92-3.18 ; \mathrm{P}<0.001)\end{array}$ & 14.79 & $\begin{array}{c}3.06(95 \% \mathrm{Cl}, \\
2.97-3.15 ; \mathrm{P}<0.001)\end{array}$ & 5.61 & $\begin{array}{c}4.27(95 \% \mathrm{Cl}, \\
3.92-4.65 ; \mathrm{P}<0.001)\end{array}$ \\
\hline
\end{tabular}

†, compared to low SDI. SDI, socio-demographic index; DALYs, disability-adjusted life years; OR, odds ratio; 95\% CI, 95\% confidence interval.

predictive and prognostic factors, including measurable biomarkers (11), by improving public awareness on the clinical, psychological, societal and economic consequence of living with LBP (12), and by developing new therapeutic interventions, either pharmacologic or physical, that would enable an earlier, stable and more efficient pain relief, especially in patients with chronic disease $(13,14)$.

As regards the sex distribution, we confirm earlier reports that women tend to suffer more of LBP than men (7). We also showed that the impact of LBP on healthy life is substantially magnified in women, since the DALYs are over $40 \%$ higher in the female sex. The data on age distribution of LBP garnered from the GHDx also provide interesting evidence. According to previous reports, LPB has been for long considered the most common type of musculoskeletal pain in older adults (15). Our analysis only partially confirms this evidence. Although the percent of total number of LBP cases recorded in each age group is mostly concentrated between 35-64 years, the worldwide prevalence grows in parallel with ageing, up to $21 \%$ after the age of 80 years. This would indeed confirm that LBP is very frequent in the elderly, but previous analyses may have probably overrated its burden in comparison to other disabling severe and/or disorders in older adults. In fact, our data shows that the relative incidence $\left(9^{\text {th }}\right.$ worldwide cause), prevalence $\left(14^{\text {th }}\right.$ worldwide cause) and DALYs $\left(12^{\text {th }}\right.$ worldwide cause) of this condition are not exceptionally different after the age of 80 years from those in the entire age range, and in no case LBP would be comprised between the 5 most frequent or debilitating diseases in the elderly.
The last important information than can be garnered from our analysis is that a direct relationship exists between the burden of LBP and SDI. We found that there is still an enormous gap, comprised between 3-4 folds, between incidence, prevalence and DALYs of LBP in high and low SDI countries. This aspect seems rather controversial in the current scientific literature. An earlier critical review of the scientific literature published in 2011 identified 17 studies which investigated the burden of LPB across different socio-economic positions, concluding that no clear relationship exists between LBP and sociodemographic characteristics (16). Later on, Chou et al. surveyed 24,435 Taiwanese adults, $26 \%$ of whom reporting at least one episode of LPB within the past 3 months, and found that low education was a significant predisposing factor for this condition (OR, 1.38; 95\% CI, $1.23-$ 1.55) (17). Finally, Stewart Williams et al. analyzed the data of the World Health Organization (WHO) Study on global AGEing and adult health (SAGE), totaling 30,146 subjects in China, Ghana, India, Mexico, South Africa and the Russian Federation, and concluded that lower education was a significant predictor of past-month LBP (18). This evidence recently led Hartvigsen and colleagues to conclude that disabling LBP may be overrepresented in countries with low socioeconomic status (8). Although we cannot actually find a reliable explanation for the discrepancy with our findings, we hypothesize that limited access to care and higher burden of more severe conditions (e.g., infections) in lower SDI countries (i.e., low and low-middle SDI) may perhaps contribute to a 
significant underdiagnosis of LBP.

\section{Conclusions}

In conclusion, the results of our analysis attest that LBP poses a significant worldwide epidemiologic burden, especially in women, adults and high SDI countries, displaying an escalating trend that is not expected to reverse soon.

\section{Acknowledgments}

Funding: None.

\section{Footnote}

Conflicts of Interest: All authors have completed the ICMJE uniform disclosure form (available at http://dx. doi. org/10.21037/jhmhp-20-17). The authors have no conflicts of interest to declare.

Ethical Statement: The authors are accountable for all aspects of the work in ensuring that questions related to the accuracy or integrity of any part of the work are appropriately investigated and resolved. The study was performed in accordance with the Declaration of Helsinki, under the terms of relevant local legislation. The data in this article were taken from a public database, so ethical review is exempt.

Open Access Statement: This is an Open Access article distributed in accordance with the Creative Commons Attribution-NonCommercial-NoDerivs 4.0 International License (CC BY-NC-ND 4.0), which permits the noncommercial replication and distribution of the article with the strict proviso that no changes or edits are made and the original work is properly cited (including links to both the formal publication through the relevant DOI and the license). See: https://creativecommons.org/licenses/by-nc-nd/4.0/.

\section{References}

1. Koes BW, van Tulder MW, Thomas S. Diagnosis and treatment of low back pain. BMJ 2006;332:1430-4.

2. Maher C, Underwood M, Buchbinder R. Non-specific low back pain. Lancet 2017;389:736-47.

3. Ehrlich GE. Low back pain. Bull World Health Organ. 2003;81:671-6.
4. Hoy D, Brooks P, Blyth F, Buchbinder R. The Epidemiology of low back pain. Best Pract Res Clin Rheumatol 2010;24:769-81.

5. Meucci RD, Fassa AG, Faria NM. Prevalence of chronic low back pain: systematic review. Rev Saude Publica. 2015;49:1.

6. Hoy DG, Smith E, Cross M, et al. Reflecting on the global burden of musculoskeletal conditions: lessons learnt from the global burden of disease 2010 study and the next steps forward. Ann Rheum Dis 2015;74:4-7.

7. Henschke N, Kamper SJ, Maher CG. The epidemiology and economic consequences of pain. Mayo Clin Proc 2015;90:139-47.

8. Hartvigsen J, Hancock MJ, Kongsted A, et al. What low back pain is and why we need to pay attention. Lancet 2018;391:2356-67.

9. Institute for Health Metrics and Evaluation. Global Health Data Exchange. Accessed, January 23, 2020. Available online: http://ghdx.healthdata.org/gbd-results-tool.

10. GBD 2017 Disease and Injury Incidence and Prevalence Collaborators. Global, regional, and national incidence, prevalence, and years lived with disability for 354 diseases and injuries for 195 countries and territories, 1990-2017: a systematic analysis for the Global Burden of Disease Study 2017. Lancet 2018;392:1789-858.

11. Lippi G, Dagostino C, Buonocore R, et al. The serum concentrations of leptin and MCP-1 independently predict low back pain duration. Clin Chem Lab Med 2017;55:1368-74.

12. Raciborski F, Gasik R, Kłak A. Disorders of the spine. A major health and social problem. Reumatologia 2016;54:196-200.

13. Foster NE, Anema JR, Cherkin D, et al. Prevention and treatment of low back pain: evidence, challenges, and promising directions. Lancet 2018;391:2368-83.

14. Shipton EA. Physical Therapy Approaches in the Treatment of Low Back Pain. Pain Ther 2018;7:127-37.

15. Makris UE, Higashi RT, Marks EGet al. Physical, Emotional, and Social Impacts of Restricting Back Pain in Older Adults: A Qualitative Study. Pain Med 2017;18:1225-35.

16. Adamson J, Hunt K, Nazareth I. The influence of sociodemographic characteristics on consultation for back pain-a review of the literature. Fam Pract 2011;28:163-71.

17. Chou YC, Shih CC, Lin JG, et al. Low back pain associated with sociodemographic factors, lifestyle and osteoporosis: a population-based study. J Rehabil Med 
2013;45:76-80.

18. Stewart Williams J, Ng N, Peltzer K, et al. Risk Factors and Disability Associated with Low Back Pain in Older

doi: $10.21037 /$ jhmhp-20-17

Cite this article as: Mattiuzzi C, Lippi G, Bovo C. Current epidemiology of low back pain. J Hosp Manag Health Policy 2020;4:15.
Adults in Low- and Middle-Income Countries. Results from the WHO Study on Global AGEing and Adult Health (SAGE). PLoS One 2015;10:e0127880. 